\title{
Von Hippel-Lindau Syndrome- A Case Report.
}

\author{
Suraj Thulung ${ }^{1}$, Ashish Baniya ${ }^{2}$, Subodh Sharma Paudel ${ }^{2}$, Anirudra Devkota ${ }^{2}$, Shikher Shrestha ${ }^{3}$ \\ ${ }^{1}$ Associate Professor, ${ }^{2}$ Medical Officer, ${ }^{3}$ Consultant Neurosurgeon, Department of Neurosurgery, Upendra Devkota Memorial \\ National Institute of Neurological and Allied Sciences, Nepal.
}

Received: July 20, 2020

Accepted: August 15, 2020

Published: September 1, 2020

Cite this paper:

Thulung S, Baniya A, Paudel SS, Devkota A, Shrestha S. Von Hippel-Lindau Syndrome- A Case Report. Journal of Brain and Spine Foundation Nepal. 2020;1(1):25-28.

Correspondence:

Suraj Thulung

Associate Professor

Department of Neurosurgery

Upendra Devkota Memorial National Institute of Neurological and Allied Sciences, Nepal.

E-mail address: surajthulung@yahoo.com

ORCID: https://orcid.org/0000-0001-5559-895X

\begin{abstract}
:
Von Hippel-Lindau (VHL) disease is a rare, familial disorder involving multiple organs, and characterized by the development of many benign and malignant tumors. It is an autosomal dominant disorder with high penetrance and variable expression. We present a case of a 31-year-old gentleman with gradual onset painless bilateral diminution in the vision for one and a half months with significant positive family history. Radiographic study revealed multiple cerebellar hemangioblastomas with obstructive hydrocephalus, renal cortical and pancreatic cysts and cystic renal cell carcinoma. Symptoms were relieved after endoscopic third ventriculostomy. These constellations of findings suggested von Hippel Lindau (VHL) syndrome and we present this case.
\end{abstract}

Key words: Hemangioblastoma, von Hippel-Lindau Disease, Ventriculostomy, Vision.

\section{Introduction:}

Von Hippel-Lindau (VHL) disease is a rare, familial disorder involving multiple organs, and characterized by the development of many benign and malignant tumors. It is an autosomal dominant disorder with high penetrance and variable expression. This occurs because of the germ-line mutation of VHL tumor suppressor gene on the short arm of the third chromosome (3p25-26). ${ }^{1}$ The diagnostic criteria for VHL disease are: (a) more than one CNS hemangioblastoma, (b) one CNS hemangioblastoma and visceral manifestations of VHL disease, and (c) any manifestation and a known family history of VHL disease. $^{2}$ We report a rare case of Von Hippel-Lindau syndrome which was symptomatically managed with endoscopic surgery and planned for further surgical evaluation.

\section{Case Summary:}

A 31-year-old gentleman presented with complaints of gradual, painless, diminished vision of the bilateral eye for one and a half months. He also complained of a gradually progressive, global headache for five months with increased severity in the early morning. He was recently diagnosed as a case of type II Diabetes mellitus. He also reported that similar symptoms were present in his brother and sister. At presentation, he was well oriented to time place and the person and his vital 
parameters were stable. His visual acuity was $6 / 36$ on right and 2/60 on left with an impaired color vision of bilateral eye $(0 / 17)$. He also found to have absolute scotoma on the left eye and central relative scotoma on right. All of his hematological and biochemical investigations were within normal limits except increased hemoglobin levels of $20 \mathrm{mg} / \mathrm{dl}$ and fasting blood glucose of $197 \mathrm{mg} / \mathrm{dl}$.

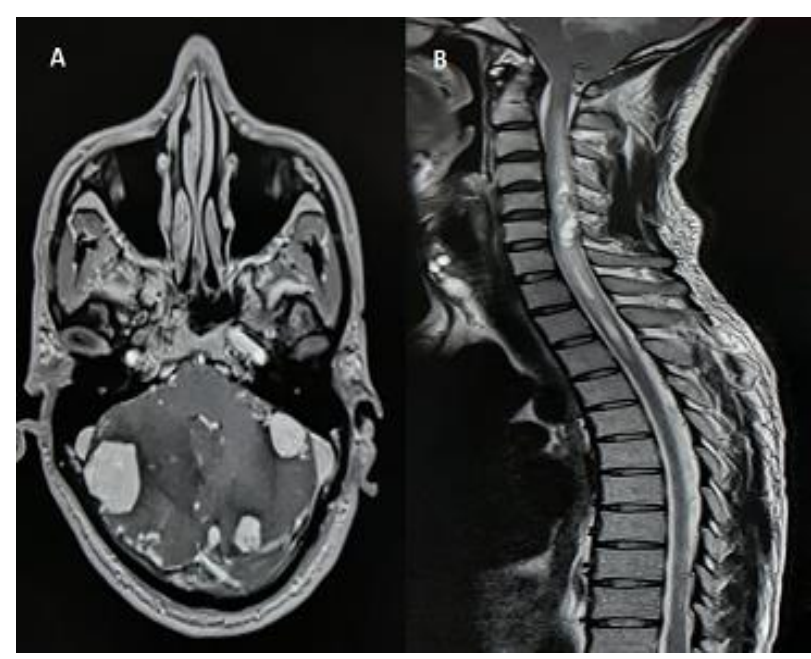

Figure 1(A) Contrast MRI brain axial view showing multiple ring-enhancing lesion on the cerebellum. Figure 1(B) Contrast MRI spine sagittal view showing multiple ring-enhancing lesions along the spinal cord.

MRI of the brain showed multiple pial based extra-axial lesions on the bilateral cerebellar hemisphere with prominent arterial feeders, marked homogenous postcontrast enhancement and mass effect, suggestive of multiple hemangioblastomas with obstructive hydrocephalus. (Figure 1A). T2 screening of the whole spine in MRI showed multi-cystic lesion intramedullary at C4 to C6 level likely hemangioblastoma with cord edema at C2 to D2 levels. (Figure 1B). CT scan of the abdomen and pelvis showed multiple innumerable variable-sized pancreatic cysts largest measuring $7 \mathrm{~cm} \mathrm{X}$ $7 \mathrm{~cm}$ in the pancreas's body. Bilateral multiple renal cortical cysts. 5.4 X $4.3 \mathrm{~cm}$ cystic lesion in left lower pole with multiple enhancing septations suggestive of Cystic Renal Cell Carcinoma (Figure 2).

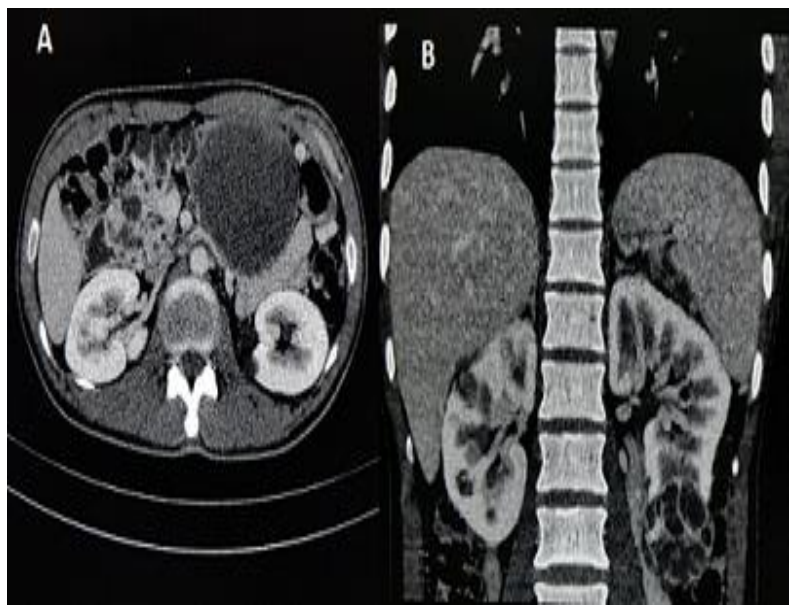

Figure 2: Contrast CT abdomen and pelvis (A) axial view and (B) coronal view showing multiple cystic lesions around the pancreas and bilateral kidney.

The patient underwent endoscopic third ventriculostomy (ETV) for obstructive hydrocephalus on the 3rd day of admission. The patient was symptomatically better after the procedure. The patient was under strict glycemic control and was managed with subcutaneous insulin as advised by treating endocrinologist. Post ETV, patient improved well in terms of his visual symptoms and headache. He was refered to a general surgeon for his abdominal findings.

\section{Discussion:}

Von- Hippel Lindau is a rare autosomal dominant disorder that occurs with a frequency of 1 per 36000 of the population and results in the development of hemangioblastoma of the Central Nervous System (CNS), Renal cell carcinoma (RCC), renal and pancreatic cyst along with pheochromocytoma. CNS hemangioblastomas are the most common tumors in VHL syndrome affecting $60-80 \%$ of patients. ${ }^{2}$ This is the major cases cause of morbidity and mortality due to mass effect on nearby structures. These hemangioblastomas can be found in the cerebellum 
(16-69\%), brainstem (5-22\%), spinal cord (13-53\%), cauda equina $(11 \%)$, or supratentorial region $(1-7 \%){ }^{3}$ The most common sites for hemangioblastoma development are the cerebellum and spinal cord as evident in our patient. Hemangioblastomas are most frequently found in the temporal peripheral retina but can also be seen in the juxtapapillary region in up to $15 \%$ of patients. However, in our patient, there was no structural lesion on the retina of both eye and his eye symptoms are mostly due to rising intracranial pressure.

Hemangioblastoma of the CNS usually develops from childhood at an age of $<10$ years or early teen until the age of 30 years. ${ }^{4}$ The mean age (and ranges) of diagnosis of retinal hemangioblastoma, cerebellar hemangioblastoma, and renal cell carcinoma are 25 years (1-67), 30 years (11-78), 37 years (16-67) respectively. ${ }^{5}$ The symptoms of hemangioblastoma are caused by an expansion of tumors in intracranial space and spinal cord, however, at the time of presentation, our patient did not have any symptoms of the spaceoccupying lesion at the spinal cord. The best treatment modality for this tumor is surgical resection, in cases of large tumor burden where surgical resection is not possible, gamma knife surgery is really a good option.

Renal cysts are present in 59-63\% of the individual in VHL and Renal cell carcinoma develops in $24-45 \%$ of VHL patients. ${ }^{5,6}$ Renal involvement in VHL is multicentric and bilateral in at least $75 \%$ of the patients. $^{7}$ Pancreatic cystadenomas or cysts are generally asymptomatic, characteristically seen as multiple cysts in the pancreas on imaging which is seen in our patient too.

This syndrome should be considered in any patient with early-onset or multifocal RCC or RCC in combination with any of the following: a history of visual or neurologic symptoms; a family history of blindness, central nervous system tumors, or renal cancer; or coexistent pancreatic cysts, epididymal lesions, or inner-ear tumors. ${ }^{8-10}$ Patients suspected of having von Hippel-Lindau disease, or the appropriate relatives of those with documented disease, should strongly consider genetic evaluation.

Recent clinical trials have been conducted with the use of anti-angiogenic therapies to stop both the initiation and progression of these highly vascular tumors seen in VHL disease. The prognosis of VHL disease initially was ascribed in accordance with the outcome of RCC treatment, but with the advent of nephron-sparing surgery and radiofrequency ablation, it is now becoming a curable condition.

\section{Conclusion:}

Being a rare disorder, VHL can present as a diagnostic dilemma to the clinicians. This requires meticulous history taking and clinical examination and multi-organ radiological evaluation. The primary site of presentation is the neurological symptoms due to mass lesions present in the brain and spinal cord. Similarly, it can present as an endocrine abnormality due to the involvement of the pancreas and adrenal medulla. Symptomatic management is the key and various new therapies like selective embolization of blood vessels are going to play a vital role in decreasing symptoms.

\section{References:}

1. Latiff F, Tory K, Gnarra J, Yao M, Duh F, Orcutt ML, et al. Identification of the von Hippel-Lindau disease tumor suppressor gene. Science. 1993; 260(5112):1317-20. https://doi.org/10.1126/science.8493574.

2. Kanno H, Kuratsu JI, Nishikawa R, Mishima K, Natsume A, Wakabayashi T, et al. Clinical features of patients bearing central nervous system hemangioblastoma in von Hippel-Lindau disease. Acta Neurochir. $2013 \quad$ Jan; 155(1):1-7. https://doi.org/10.1007/s00701-012-1514-y.

3. Varshney N, Kebede AA, Owusu-Dapaah H, Lather J, Kaushik M, Bhullar JS. A review of Von Hippel-Lindau 
syndrome. J Kidney Cancer VHL. 2017; 4(3):20-9. https://doi.org/10.15586/jkcvhl.2017.88.

4. Shuin T, Yamasaki I, Tamura K, Okuda H, Furihata M, Ashida S. Von Hippel-Lindau disease: molecular pathological basis, clinical criteria, genetic testing, clinical features of tumors and treatment. Jpn $\mathbf{J}$ Clin Oncol. 2006 Jun; 36(6):337-43. https://doi.org/10.1093/jjco/hyl052.

5. Maher ER, Yates JR, Harries R, Benjamin C, Harris R, Moore AT, et al. Clinical features and natural history of von Hippel-Lindau disease. Q J Med. 1990;77(283):1151-1163. https://doi:10.1093/qjmed/77.2.1151.

6. Maher ER, Iselius L, Yates JR, Littler M, Benjamin C, Harris R, et al. Von Hippel-Lindau disease: a genetic study. J Med Genet. 1991 Jul; 28(7):443-7. https://doi.org/10.1136/jmg.28.7.443.

7. Quadery FA, Okamoto K. Diffusion-weighted MRI of haemangioblastomas and other cerebellar tumours. Neuroradiology. $2003 \quad$ Apr; 45(4):212-9. https://doi.org/10.1007/s00234-003-0951-y.

8. Neumann HP, Zbar B. Renal cysts, renal cancer and von Hippel-Lindau disease. Kidney Int. 1997 Jan;51(1):1626. https://doi.org/10.1038/ki.1997.3.

9. Linehan WM. Molecular targeting of VHL gene pathway in clear cell kidney cancer (editorial). J Urol.2003 Aug;170(2):593-4. https://doi.org/10.1097/01.ju.0000077210.05543.a.

10. Choyke PL, Glenn GM, Walther MM, Zbar B, Linehan WM. Hereditary renal cancers. Radiology. 2003 Jan; 226(1):33-46.

https://doi.org/10.1148/radiol.2261011296. 